\title{
Building an IT Infrastructure for Citizen Science Research on Climate Change
}

\author{
Anudari Batsaikhan ${ }^{1}$ \\ Leibniz Supercomputing Centre (LRZ) of the Bavarian Academy of Science and Humanities \\ Boltzmannstraße 1, 85748 Garching bei München, Germany \\ E-mail: anudari.batsaikhanelrz.de
}

\author{
Jens Weismüller \\ Leibniz Supercomputing Centre (LRZ) of the Bavarian Academy of Science and Humanities \\ Boltzmannstraße 1, 85748 Garching bei München, Germany \\ E-mail: jens.weismuellerelrz.de
}

\section{Stephan Hachinger}

Leibniz Supercomputing Centre (LRZ) of the Bavarian Academy of Science and Humanities Boltzmannstraße 1, 85748 Garching bei München, Germany

E-mail: stephan.hachingerelrz.de

Due to the far-reaching consequences of climate change, extensive adaptation and climate protection measures are becoming necessary not only on a global, but in particular on more regional scales. Such measures need to embrace citizens for their success, on an educational as well as a participatory level. For Bavaria, Germany, the BAYSICS project (Bavarian Citizen Science Portal for Climate Research and Science Communication) aims to achieve (1) innovative digital forms of citizens' participation in climate change research, (2) transfer of knowledge on the complexity of climate change and its local consequences, and (3) joint scientific and environmental education goals.

Within the project we develop two main tools for citizens, an interactive web portal and smartphone app. As the project processes data collected by citizens, careful considerations are necessary, especially on legal issues (e.g. copyright), the privacy of citizens, and the credibility of data. Data collected by the citizens are visualized on the interactive web portal. Additionally, the data will be available for download through the web portal. Thus, clear metadata (e.g. on relevant attributes and data usage license) needs to be included. To increase the credibility of data, data collection guideline for citizens and the application of trust metrics to data are considered.

International Symposium on Grids \& Clouds 2019, ISGC2019

31st March - 5th April 2019

Academia Sinica, Taipei, Taiwan 


\section{Introduction}

Climate change has wide-ranging consequences on all scales, ranging from the global to regional and local scales [1]. In Germany, the strong trend of warming has been observed in the Southwest and in the mountain areas [2]. As the impacts vary geographically, adaptation and climate protection measures are necessary particularly on the regional scale $[1,2]$.

Successful implementation of these measures requires citizens' understanding and participation. Citizen science covers both the educational and participatory aspects. Citizens are able to choose their research topics by themselves or specific topics can be used in school/university projects. The data is collected by the participants $[3,4]$. From the educational aspect, citizens' knowledge and awareness towards the environment is increased through experiential learning [3, 4]. From the participatory aspect, citizens take part in scientific activities and provide data [3], and citizens' engagements e.g. in environment conservation actions can be fostered [4].

Climate change education and awareness have gained recognition over the years as a critical element to deal with climate change. The United Nations have adopted various programs to support actions on climate change education, training, public awareness, public participation and access to information [5]. Such actions are essential to promote sustainable development under climate change. The advancement of digital technologies has enabled environmental education on climate change through digital platforms $[6,7]$. While each of these technologies has its own benefits and challenging aspects, they can provide effective and innovative learning opportunities [8]. For the future, citizen science with the use of digital technologies is expected to play an important role to achieve the Sustainable Development Goals 2030 [9].

Citizen science is globally taking place in the fields such as public health, disaster risk assessment, and ecology (e.g. HealthMap, Mosquito Alert, Safecast, and Nature Watch). At the European level, the European Citizen Science Association (ECSA) works as the network which supports citizen science initiatives. In Germany, the "Bürger schaffen Wissen" is the central platform for citizen science projects where for e.g. more than 60 projects were registered in 2016 [10]. In their strategy paper 2020, Bonn et al. (2016) highlighted the importance of collaboration across science, society and politics, and participation through web-based infrastructures for active knowledge exchange [10].

Opinions differ about the quality of data collected by citizens. However, there is evidence that these data are useful to study climate change [11-13]. It has been shown that the quality in some cases can keep up with data collected by professionals $[14,15]$. Yet there are concerns remaining [16]. In our BAYSICS project (introduced below) data collected by the citizens is one of the key parts. The IT infrastructure gives researchers the opportunity to integrate the data collection guidelines for citizens, design the input data fields, acquire the data through the database, and assess the collected data using their own methods (e.g. application of trust metrics).

In this work, the IT infrastructure for the BAYSICS project is described. BAYSICS (Bavarian Citizen Science Portal for Climate Research and Science Communication, 2018-2023) is a scientific project which promotes citizens' research on climate change in Bavaria, Germany. The project aims to achieve (1) citizens' active participation in research on climate change through digital platforms, (2) transfer of knowledge on the complexity of climate change and its local consequences, and (3) joint scientific and environmental education goals. It has 10 partner institutions with researchers in natural sciences, environmental education, and environmental sociology. In the natural science field, it has four research topics (phenology, pollen, tree heights, and animals). 
An interactive web portal and a smartphone app are developed as platforms for citizens. The data will be collected by citizens under the four topics (plant types and their growth stages, pollen allergy symptoms, tree types and heights, and number of animals and their activities observed) mainly through the smartphone app, and their data will be processed, shown and visualized on the web portal. The collected data will also be used by researchers for scientific purposes. The web portal and the smartphone app will be tested and implemented at educational institutions. The project started in 2018 and is currently in the development phase. The IT infrastructure was designed based on the needs/aims of the project and has been developed as introduced in the next section.

\section{IT Infrastructure}

\subsection{Overview}

The IT infrastructure (Figure 1) consists of a frontend server (Application Server) and a backend server holding the database (Database server). The frontend makes data visible to users via a web/mobile app and allows users to submit the observation data. The backend serves for data storage and may also be queried directly for scientific data evaluation.

The infrastructure relies on open-source tools as shown in Figure 1. For the database, PostgreSQL and PostGIS are chosen. The web portal is developed in Python using the Django web framework [e.g. 17-19]. A REST API is written to connect the developed framework with a smartphone App.

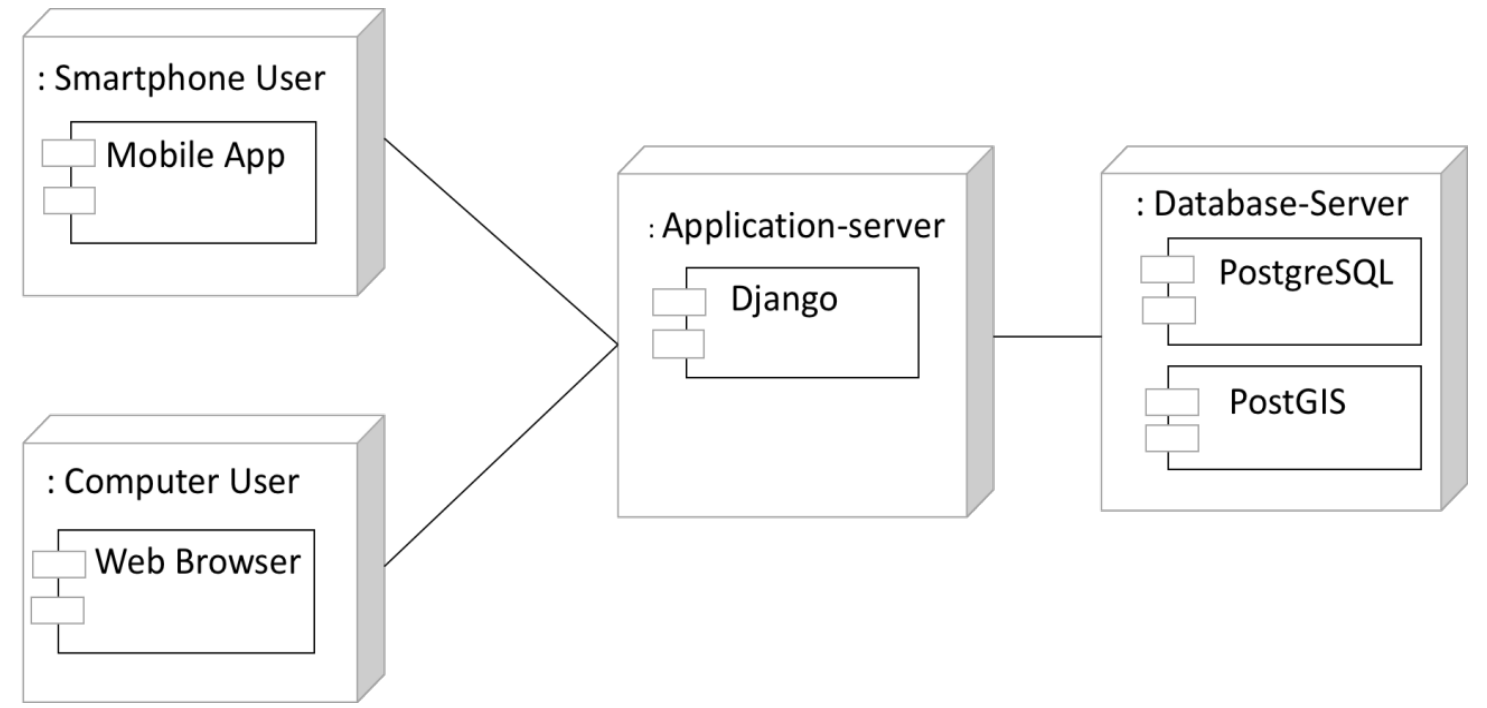

Figure 1. Simplified diagram of the IT architecture which illustrates the main components (the users, the application server, and the database server) and their relationships. The left side shows the users, the middle application server, and the right side the database server. 


\subsection{Database}

A database which stores information about the users and their observations was developed to suit the needs of the project.

To submit observations in the platform, a user needs to sign-up and log into the platform. User authentification and authorization related information (e.g. username, email address, and password) is stored in a data table (" 1 " in Figure 2). For storing passwords, a hashing system is applied, which was provided in the web framework used for this project. Since the demographic profile of a user (e.g. gender and age group) is also of interest within the project, a data table to store such information was created ("2" in Figure 2). It is optional for a user to provide such profile information.

In the central data table (" 3 " in Figure 2), general information about the observations, which were required in all research topics, is stored. For example, geographical location, date and time of the observations are included in this data table. Research-topic specific data tables were created, as each research topic (c.f. the section 1) has different questions about the observations ("4" in Figure 2).

A user is able to edit own observation entries and delete the user account. When a user account is deleted, the observations provided are still kept in the database, but assigned to "an anonymous user". Overall, the user identifier (" 1 " in Figure 2) is used as little as possible when establishing table relations in order to keep the observation data apart from the user related information for user's privacy.

For all research topics, users are asked to submit photos of their observations (vegetation and animals observed). Storage for such photos is in preparation.

4

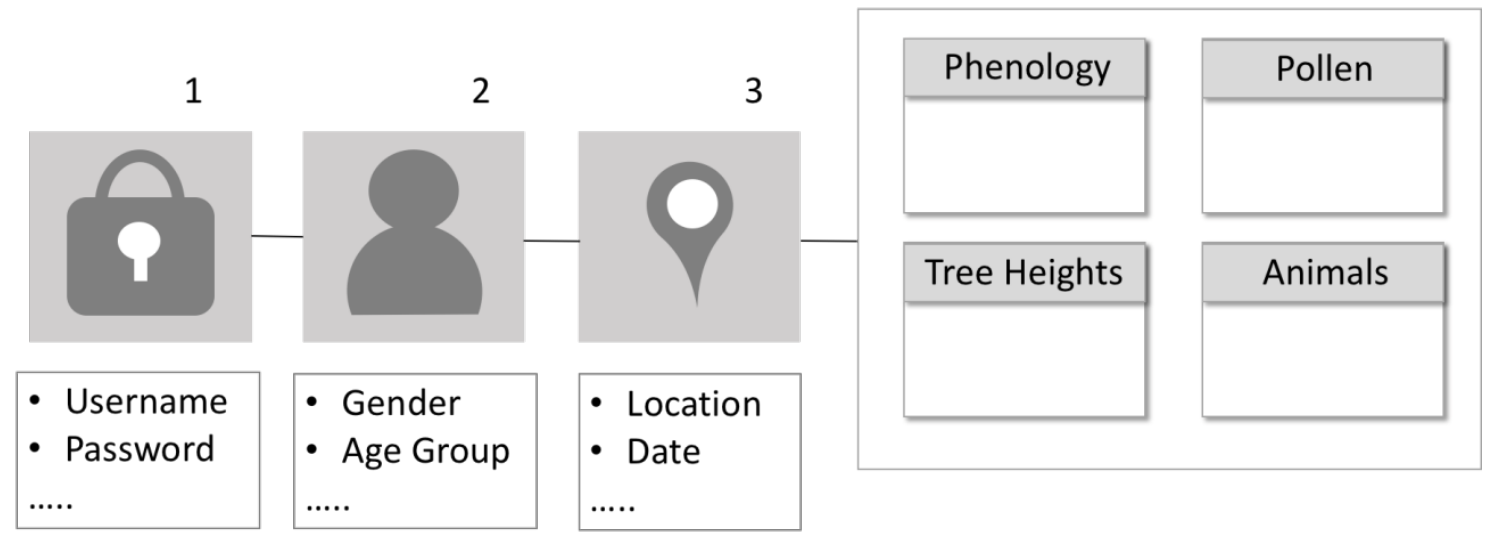

Figure 2. Data table architecture (simplified). In Table "1" the user authentification and authorization-related information is stored. Table "2" contains the demographic profile. The observation related information is stored in Table " 3 " and in Table " 4 ". 
For users who want to submit the observation data the user registration is mandatory to secure the quality of the data which goes into the database and for providing the possibility of customization for users. Thus, users are able to review their observations and edit them after their submissions. The table architecture (Figure 2) can be devided in two parts, into the user related information and the observation related information. It was designed in a way that it follows the steps of user inputs and that researchers have easy access to the information from each step.

\section{Future work in the project}

The observation data within the project will be freely available for download on the project's web portal. It aims to make the data as "FAIR (Findable, Accessible, Interoperable, Reusable)" as possible [20]. Thus, an appropriate metadata with relevant attributes as well as a data licensing scheme need to be prepared for this purpose. The data for download will not include any user related information to avoid tracking or identification of a user. The prototype of the web portal/smartphone app will be tested at educational institutions. A knowledge base and e-learning module will be included in the project's platforms, supporting educational purposes.

\section{Summary}

The IT platform of the BAYSICS project supplies basic infrastructure for citizen science research on climate change. To build up this platform, the needs/aims of the project were first identified and then integrated.

The IT infrustructure relies entirely on open-source tools such as Django, PostgreSQL, and PostGIS. The database which stores the user-related information and their observations consists of conventional database tables, but also of additional components such as storage for photos taken by the citizens. The data collected within the project will be freely available for download on the project's web portal, supporting the FAIR principles.

\section{Acknowledgement}

The BAYSICS project is sponsored by the Bavarian State Ministry of Science and the Arts in the context of the Bavarian Climate Research Network (bayklif).

\section{References}

[1] The Intergovernmental Panel on Climate Change (IPCC), Summary for Policymakers, in Global Warming of $1.5^{\circ} \mathrm{C}$ - An IPCC Special Report on the impacts of global warming of $1.5^{\circ} \mathrm{C}$ above preindustrial levels and related global greenhouse gas emission pathways, in the context of strengthening the global response to the threat of climate change, sustainable development, and efforts to eradicate poverty, V. Masson-Delmotte, P. Zhai, H.-O. Pörtner, D. Roberts, J. Skea, P.R. Shukla, A. Pirani, W. 
Moufouma-Okia, C. Péan, R. Pidcock, S. Connors, J.B.R. Matthews, Y. Chen, X. Zhou, M.I. Gomis, E. Lonnoy, Maycock, M. Tignor, and T. Waterfield (eds.), World Meteorological Organization, Geneva, Switzerland, 2018.

[2] M. Zebisch, T. Grothmann, D. Schröter, C. Hasse, U. Fritsch, and W. Cramer, Climate change in Germany - Vulnerability and adaptation of climate sensitive sectors, Federal Environmental Agency 2005.

[3] B. Zoellick, S.J. Nelson, and M. Schauffler, (2012) Participatory science and education: bringing both views into focus, Frontiers in Ecology and the Environment 10(6).

[4] H.L. Ballard, C.G.H. Dixon, and E.M. Harris, (2017) Youth-focused citizen science: Examining the role of environmental science learning and agency for conservation, Biological Conservation 208.

[5] The United Nations Educational, Scientific and Cultural Organization (UNESCO) and the secretariat of the United Nations Framework Convention for Climate Change (UNFCCC), Actions for climate empowerment - guidelines for accelerating solutions through education, training, and public awareness, UNESCO 2016.

[6] J. Coelho, A. Teixeira, P. Nicolau, S. Caeiro, and V. Rocio, (2015) iMOOC on Climate Change: Evaluation of a Massive Open Online Learning Pilot Experience, The International Review of Research in Open and Distributed Learning, 16(6).

[7] J.J. Lee, P. Ceyhan, W. Jordan-Cooley, and W. Sung, (2013) Greenify: A real-world action game for climate change education, Simulation \& Gaming 44(2-3).

[8] A. Alexandu, M. Ianculescu, E. Tudora, and O. Bica, (2013) ICT challenges and issues in climate change education, Studies in Informatics and Control 22(4).

[9] United Nations, Chapter 3 Perspectives of scientists on technology and the SDGs, in Global Sustailable Development Report 2016, UN 2016.

[10] A. Bonn, A. Richter, K. Vohland, L. Pettibone, M. Brandt, R. Feldmann, C. Goebel, C. Grefe, S. Hecker, L. Hennen, H. Hofer, S. Kiefer, S. Klotz, T. Kluttig, J. Krause, K. Küsel, C. Liedtke, A. Mahla, V. Neumeier, M. Premke-Kraus, M.C. Rillig, O. Röller, L. Schäffler, B. Schmalzbauer, U. Schneidewind, A. Schumann, J. Settele, K. Tochtermann, K., Tockner, J. Vogel, W. Volkmann, H. von Unger, D. Walter, M. Weisskopf, C. Wirth, T. Witt, D. Wolst, and D. Ziegler, Green Paper Citizen Science Strategy 2020 for Germany, Helm- holtz Centre for Environmental Research (UFZ), German Centre for integrative Biodiversity Research (iDiv) Halle-Jena-Leipzig, Leipzig, Museum für Naturkunde Berlin, Leibniz Institute for Evolution and Biodiversity Science (MfN), BerlinBrandenburg Institute of Advanced Biodiversity Research (BBIB), Berlin 2016.

[11] A.H. Hurlbert, and Z. Liang, (2012) Spatiotemporal variation in avian migration phenology: citizen science reveals effects of climate change. PLoS One 7(2).

[12] G.A. Weyhenmeyer, M. Mackay, J.D. Stockwell, W. Thiery, H-P. Grossart, P.B. Augusto-Silva, H.M. Baulch, E. de Eyto, J. Hejzlar, K. Kangur, G. Kirillin, D.C. Person, J.A. Rusak, S. Sandro, and R. lestyn Woolway, (2017) Citizen science shows systematic changes in the temperature difference between air and inland waters with global warming. Scientific Report 7(43890). 
[13] J.T. Snyder, M.M. Whitney, H.G. Dam, M.W. Jacobs, and H. Baumann, (2019) Citizen science observations reveal rapid, multi-decadal ecosystem changes in eastern Long Island Sound, Marine Environmental Research (In Press).

[14] A.W. Crall, G.J. Newman, T.J. Stohlgren, K.A. Holfelder, J. Graham., and D.M. Waller, (2011) Assessing citizen science data quality: An invasive species case study. Conservation Letters 4(6).

[15] M. Kosmala, A. Wiggins, A. Swanson, and B. Simmons, (2016) Assessing data quality in citizen science. Frontiers in Ecology and the Environment 14(10).

[16] H.K., Burgess, L.B. DeBey, H.E. Froehlich, N. Schmidt, E.J. Theobald, A.K. Ettinger, J. HilleRisLambers, J. Tewksbury, and J.K. Parrish, (2017) The science of citizen science: Exploring barriers to use as a primary research tool, Biological Conservation 208.

[17] J.W. Bubnicki, M. Churski, and D.P.J. Kuijper, (2016) Trapper: an open source web-based application to manage camera trapping projects, Methods in Ecology and Evolution 7(10).

[18] A.S. Jones, J.S. Horsburgh, D. Jackson-Smith, M. Ramírez, C.G. Flint, and J. Caraballo, (2016) A web-based interactive visualization tool for social environmental survey data, Environmental Modelling \& Software 84.

[19] Ü. Seren, D. Grimm, J. Fitz, D. Weigel, M. Nordborg, K. Borgwardt, and A. Korte, (2017) AraPheno: a public database for Arabidopsis thaliana phenotypes, Nucleic Acids Research 45(D1).

[20] M.D. Wilkinson, M. Dumontier, I.J. Aalbersberg, G. Appleton, O. Dumon, P. Groth, M. Axton, A. Baak, N. Blomberg, J.W. Boiten, L.B. da Silva Santos, P.E. Bourne, J. Bauwman, A.J. Brookes, T. Clark, M. Crosas, I. Dillo, S. Edmunds, C.T. Evelo, R. Finkers, A. Gonzalez-Beltran, P. Rocca-Serra, S.A. Sansone, A.J.G. Gray, C. Goble, J.S. Grethe, J. Heringa, R. Kok, P.A.C. 't Hoen, R. Hooft, T. Kuhn, J. Kok, S.J. Lusher, M.E. Martone, A. Mons, A.L. Packer, B. Persson, M. Roos, M. Thompson, R. van Schaik, E. Schultes, T. Sengstag, T. Slater, G. Strawn, M.A. Swertz, J. van der Lei, E. van Mulligen, J. Velterop, A. Waagmeester, P. Wittenburg, K. Wolstencroft, J. Zhao, B. Mons, (2016) The FAIR guiding principles for scientific data managment and stewardship, Scientific Data 3 (1). 